\title{
Constraint Rules and Matching Micro-clusters Based Affinity Propagation Clustering Algorithm
}

\author{
Li-min WANG ${ }^{1}$, You ZHOU², Xu-ming HAN ${ }^{3}$, Yi-zhang WANG ${ }^{4 *}$, Jing-lin YU ${ }^{1}$, Shuai WANG \\ ${ }^{1}$ School of Internet Finance and Information Engineering, Guangdong University of Finance, \\ Guangzhou, 510521, China \\ wlm_new@163.com, yujinglin@gduf.edu.cn \\ ${ }^{2}$ College of Computer Science and Technology, Jilin University, 130012, Changchun, China \\ zyou@jlu.edu.cn \\ ${ }^{3}$ College of Information Science Technology, Jinan University, Guangzhou, 510632, China \\ hanxvming@163.com \\ ${ }^{4}$ College of Information Engineering, Yangzhou University, 225127, Yangzhou, China \\ wyzhang_new@sina.com (*Corresponding author) \\ ${ }^{5}$ Business School, Jilin University, 130012, Changchun, China \\ wang.s@qq.com
}

\begin{abstract}
The performance of original affinity propagation (AP) clustering algorithm is greatly influenced by an important parameter: preference (median of similarities between data points), and it may be difficult to identify complex structure data. To address the afore-mentioned issues, this paper proposes two novel methods namely the constraint rules-based affinity propagation (CRAP) and matching micro-clusters hierarchical clustering algorithm (MMHC). The CRAP algorithm can obtain better results by searching the optimal preference value by means of the constraint rules-based search algorithm (CRS). The MMHC algorithm initially takes results of AP as micro-clusters, then they are matched in order to achieve the right partitions of complex structure data. Experimental results demonstrate that the improved clustering algorithm performs better than AP.
\end{abstract}

Keywords: Affinity propagation, Constraint rules, Micro-clusters hierarchical clustering.

\section{Introduction}

Frey and Dueck proposed a novel clustering algorithm called affinity propagation (AP), which conducts by passing messages (Frey \& Dueck, 2007). Unlike k-means, AP begins with a large number of clusters then makes pruning decisions and it does not depend on initial center selection. The above advantages decide that AP is a better tool for data mining and pattern recognition.

At present, this method has been successfully applied to image segmentation (Zhang et al., 2017), image retrieval (Sun et al., 2013), image classification (Li et al., 2017), gene recognition (Sun et al., 2017), text clustering and many other fields (Zhang et al., 2013). Their researches extend AP's application areas.

However, it is difficult to obtain appropriate preferences (parameter of AP algorithm) values. Moreover, AP fails to deal with complex structure data because it is based on Euclidian distance similarities.

This paper proposes a parameter search algorithm and employs AP's features of identifying local dense data points to identify complex structure data. The contributions of the proposed method are as follows: the relations of internal evaluation index and clustering results are discussed, constraint rules-based affinity propagation (CRAP) is proposed to obtain optimal number of clusters. This paper discusses how to define distance of inter-class and intra-class in complex structure data, MMHC algorithm is proposed to better identify clusters with complex structure.

The remainder of this paper is as follows: Section 2 presents a brief overview of AP and its related works, while Section 3 draws the proposed constraint rules-based affinity propagation to get more promising results. Section 4 explains the proposed matching micro-clusters hierarchical clustering algorithm, while Section 5 presents experimental results using UCI and synthetic data sets. Section 6 shows the application in Chinese stock quality assessment. Finally, Section 7 concludes this paper.

\section{Related Work}

In this section, AP and its related works are reviewed. AP takes similarities between different data points as model input. For data points $x_{i}$ and 
$x_{k}$, their similarity is computed by $S(i, k)=-\left\|x_{i}-x_{k}\right\|$ (Wei et al., 2017). Later, the median of similarities is chosen as shared values, which is called preferences $(P)$ (Wang et al., 2017). Then, there are two kinds of exchanged messages deciding which points are exemplars (cluster centers) and which data point they belong to (Sun et al., 2017; Li et al., 2017). The "Responsibility" reflects how well-suited point $k$ is to be chosen as the exemplar for point $i$, the "Availability" reflects how appropriate it is for point $i$ to choose $k$ as its cent. The two kinds of messages are computed by equations (1) and (2):

$$
\begin{aligned}
& R(i, k)=S(i, k)-\max _{k^{\prime} s . t k^{\prime} \neq k}\left\{A\left(i, k^{\prime}\right)+S\left(i, k^{\prime}\right)\right\} \\
& A(i, k)=\min \left\{0, R(k, k)+\sum_{i^{\prime} s . i^{\prime} \in\{, i, k\}} \max \left\{0, R\left(i^{\prime}, k\right)\right\}\right\}
\end{aligned}
$$

To prevent the algorithm from oscillation when updating the messages, damping factor $\lambda$ is used by equation (3) and (4):

$$
\begin{aligned}
& R(i, k)^{(t+1)}=\lambda R(i, k)^{t}+(1-\lambda) R(i, k)^{(t-1)} \\
& A(i, k)^{(t+1)}=\lambda A(i, k)^{t}+(1-\lambda) A(i, k)^{(t-1)}
\end{aligned}
$$

The algorithm is terminated when cluster centers do not change for ten iterations (Fan et al., 2019). The procedure of original AP algorithm is shown in Table 1.

Table 1. Procedure of original AP algorithm

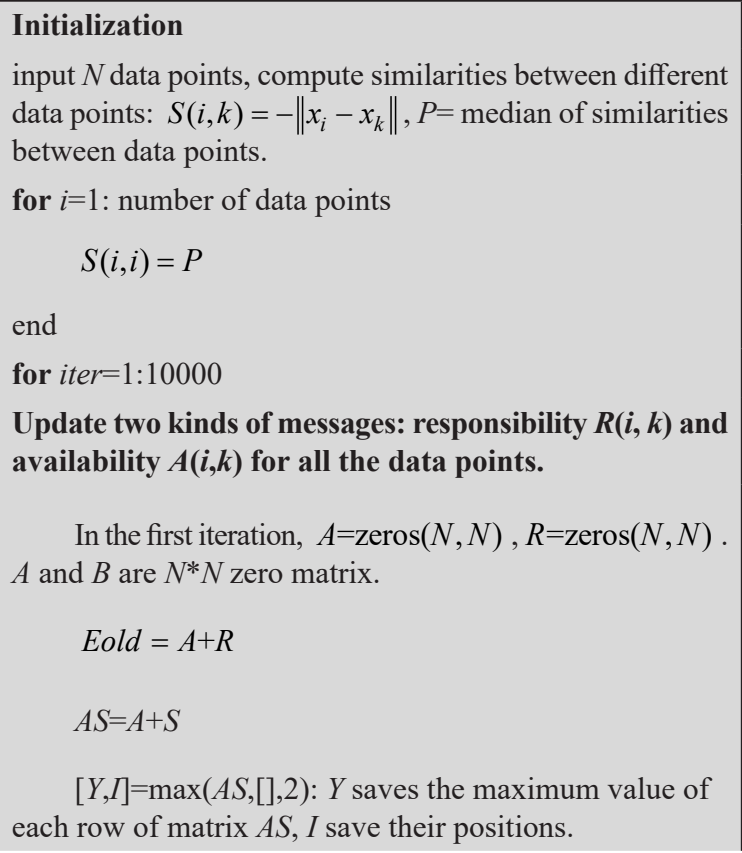

$[Y, I]=\max (A S,[], 2): Y$ saves the maximum value of each row of matrix $A S, I$ save their positions.

\section{for $i=1: N$}

$A S(i, I(i))=-$ realmax: realmax is the largest positive number in the MATLAB.

\section{end}

$[Y 2, I 2]=\max (A S,[], 2): Y 2$ saves the maximum value of each row of matrix $A S$.

$R=S$-repmat $(Y,[1, N]):$ repmat() means copying $1 * N$ matrix $Y$.

for $i=1: N$

$$
R(i, I(\mathrm{i}))=S(i, I(i))-Y 2(i)
$$

end;

$R=(1-\lambda) * R+\lambda *$ Rold

Aold $=$ A

$R p=\max (R, 0)$ : choose the larger value between each element in $R p$ and zero.

for $k=1: N$

$$
R p(k, k)=R(k, k)
$$

end;

$A=$ repmat (sum $(R p, 1),[N, 1])-R p: \operatorname{sum}(R p, 1)$ means adding each row of $R p$, than one can copy $N^{*} 1 \operatorname{sum}(R p, 1)$.

$d A=\operatorname{diag}(A): \operatorname{diag}()$ means elements on the diagonal of a matrix.

$A=\min (A, 0)$ : choose the smaller value between each element in $A$ and zero.

for $k=1: N$

$$
A(k, k)=d A(k)
$$

end

$$
A=(1-\lambda) * A+\lambda * \text { Aold }
$$

$E=A+R$

if $\operatorname{diag}($ Eold $)==\operatorname{diag}(E): \operatorname{diag}()$ means elements on the diagonal of a matrix.

count $=$ count +1

$$
\begin{aligned}
& \text { if } \text { count }==10 \\
& \text { break } \\
& \text { end }
\end{aligned}
$$$$
\text { end }
$$

end

\section{Obtain final clustering results $i d x$}

centers $=$ find $(\operatorname{diag}(E)>0)$ : the corresponding diagonal elements greater than 0 are centers.

$[\operatorname{tmp} i d x]=\max (E(:, I),[], 2)$ : each data points is assigned to the center with largest value. The $i d x$ is final clustering results. 
In recent years, scholars have developed many improving methods, all of which focus on three issues: similarity, complex data processing and application. (Gao et al., 2019) identified the problem of vulnerable line in smart grid systems by using an improved AP, which considers the topological parameters and the electrical properties (Gao et al., 2019). (Lu \& Carreira-Perpinan, 2008) developed a new constrained spectral clustering algorithm based on AP. (Zhang et al., 2013) redefined manifold similarity so as to identify complex structure data flow. (Zhang et al., 2014) proposed a hierarchical AP. Zhang X. applied AP to deal with streaming data just like (Givoni et al., 2012) applied a combination of AP and hierarchical clustering to simulate HIV strains mutation. (Qasim et al., 2013) combined AP and text concept map semi-automatic generation technique supported by experts. (Hassanabadi et al., 2014) used affinity propagation to process vehicular network.

Beside AP algorithm, there are other important clustering algorithms such as partition-based clustering (Feldman et al., 2020), density-based clustering (Darabkh et al., 2020; Angelova et al., 2020), hierarchical clustering (Dutta et al., 2020), and deep clustering (Fard et al., 2020).

Clustering is an important data mining technology in finance, economics and so on, AP can work well on them. Inspiration is drawn from the related works and then this paper proposes CRAP and MMHC for addressing these issues. For enhancing the performances of AP, CRAP is an optimal parameter method. For identifying the complex structure data, MMHC is different from (Zhang et al., 2013), which redefined manifold similarity, while MMHC constructs a graph and defines new graph similarity to measure intra-class compactness and inter-class separation.

\section{Constraint Rules based Affinity Propagation (CRAP)}

\section{Relations of Preferences $(P)$ and Number of Clusters (NC)}

Although there is no exact relation between the parameters $P$ and $N C$, one can find their fuzzy correlation. As it is shown in Figure 1 (a) (f), a synthetic dataset generated by normal distribution is given (class $1 \sim N(\mu=2, \sigma=1)$, class $2 \sim N(\mu=7$, $\sigma=1)), N C$ becomes smaller, while the absolute $P$ value increases gradually. The stable spaces, red thick line area in Figure 1(f), will naturally occur in $P-N C$ plot. High-quality clustering results (the true $N C$ ) are between these two stable spaces, but it is hard to know which stable stage is right.

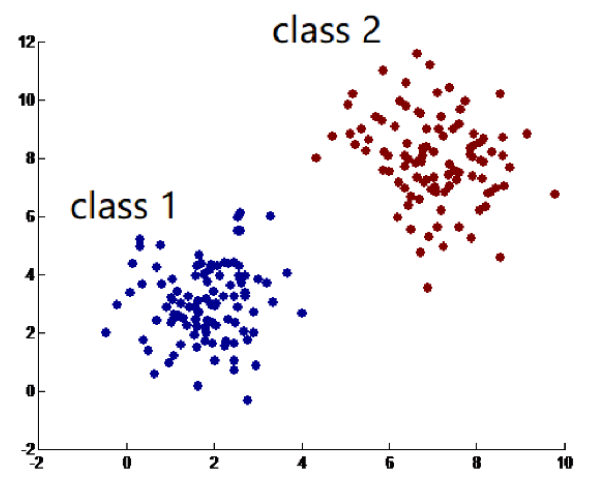

(a) ground-truth of synthetic dataset (class $1 \sim N(\mu=$ 2, $\sigma=1)$, class $2 \sim N(\mu=7, \sigma=1)$ ), different colour represents different clusters

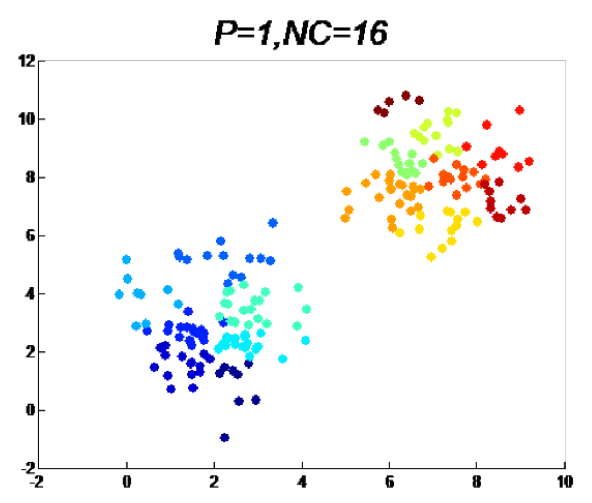

(b) $P=1$

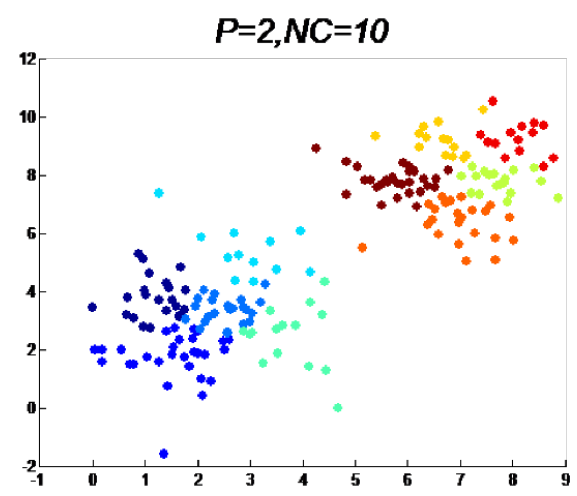

(c) $P=2$ 


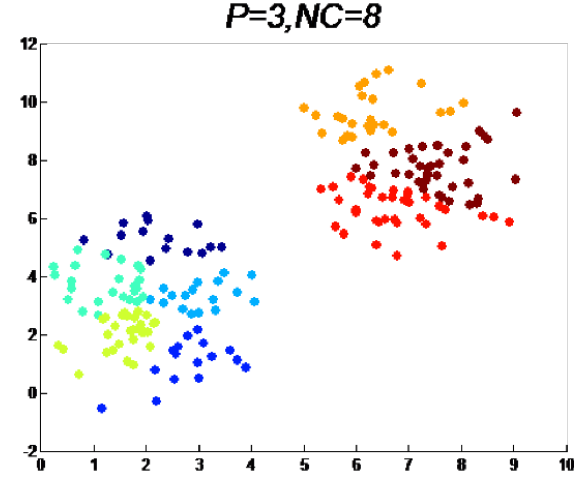

(d) $P=3$

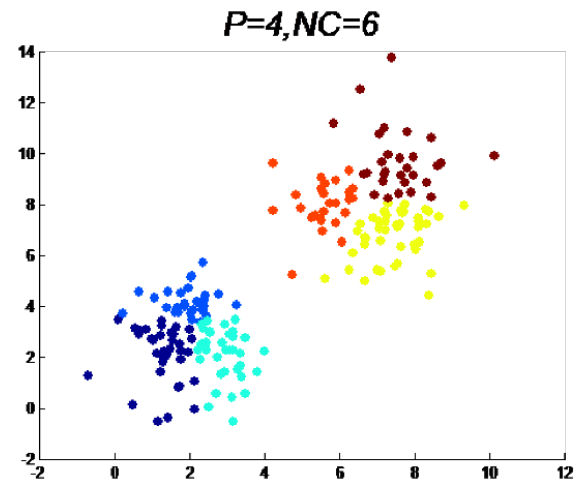

(e) $P=4$

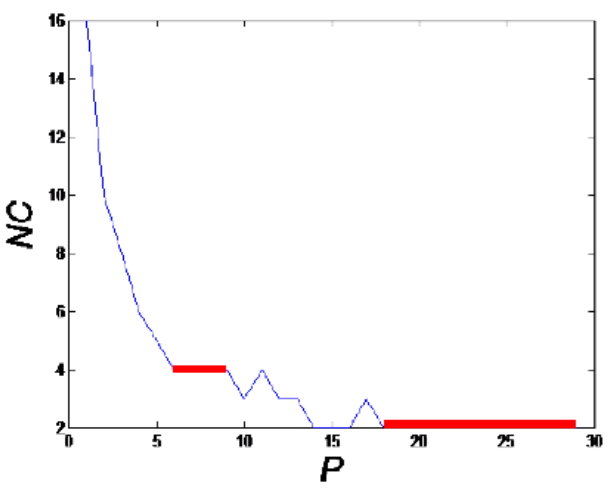

(f) $P$-NC plot

Figure 1. Relation between the parameters $P$ and $N C$ using synthetic data, which is a two-cluster dataset generated by two different kinds of normal distributions

\section{Relations of Silhouette Index (Sil) and Number of Clusters (NC)}

The popular and important internal evaluation index Silhouette (Sil) without labels is introduced to find the fuzzy relationship between number of clusters $(N C)$ generated by AP and different $P$ values. For data point $t, a(t)$ is average dissimilarity between $t$ and other data points in the same cluster, $d\left(t, C_{j}\right)$ is average dissimilarity of $t$ from cluster $C_{j}$ to other clusters $\quad C_{i}(i \neq j, i=1,2, \ldots, k), \quad b(t)=\min \left\{d\left(t, C_{j}\right)\right\}$. Every sample $t$ has its $\operatorname{Sil}(t)$ using:

$$
\begin{aligned}
& \operatorname{Sil}(t)=\frac{b(t)-\mathrm{a}(t)}{\max \{b(t), \mathrm{a}(t)\}} \\
& \text { Sil }=\operatorname{mean}\left\{\sum_{1}^{n} \operatorname{Sil}(t)\right\}
\end{aligned}
$$

Silhouette index is calculated by (6). A higher overall average $\mathrm{Sil}$ value means the clustering method performs better. According to practical experience, Sil $>0.5$ means visible boundary between different clusters and AP will oscillate when Sil $<0.2$.

Sil is used to choose stable stage in Figure 1 (e). Figure 2 shows that $P=24$ is better than $P=8$ because its Sil is bigger. In fact, Figure 2(b) is divided into two clusters, which performs better than Figure 2(a) because the true number of clusters for dataset in Figure 2 is two.

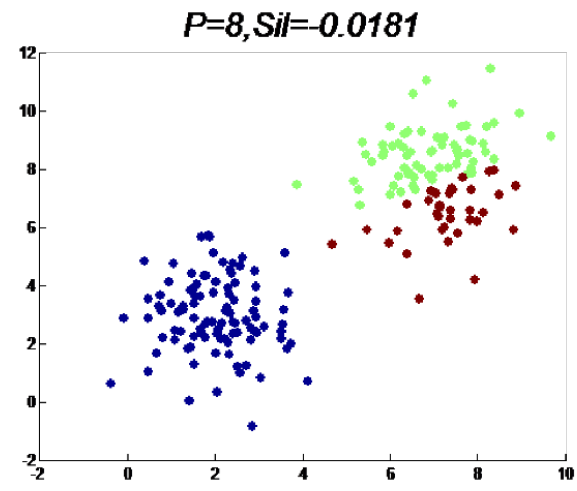

(a) $P=8$

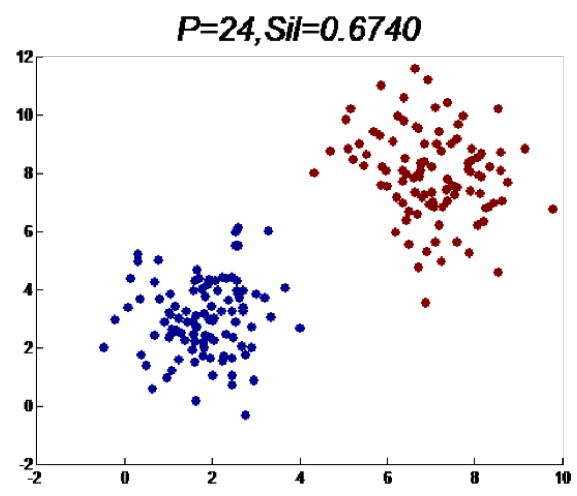

(b) $P=24$

Figure 2. Clustering results using AP with different $P$ values 
Silhouette may help one find reasonable partition of data points, so that the relationship between $\mathrm{NC}$ and Sil can be further mined out. As it can be seen, Figures 3 (a) and (b) show the effect of input preference on $N C$ and Sil using Wine and Iris, datasets between positive, continuous and steady subspace one can get optimal $N C$, subspace of Wine starts from $P_{i}=13$ and Iris is $P_{i}=12$. From relations of $\mathrm{Sil}$ and $\mathrm{NC}$, the stable space of $\mathrm{Sil}$ and $N C$ can be found, and better clustering results must be there, where exists true $N C$.

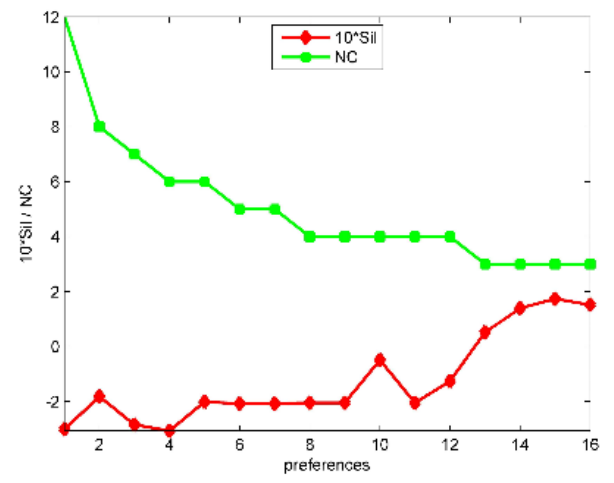

(a) The wine dataset (3-clusters dataset), $\{$ Sil / NC $\mid P \in[13,16]\}$ is the stable space

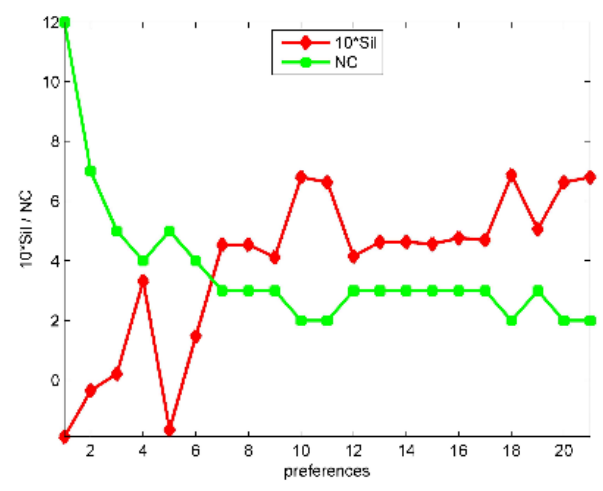

(b) The iris dataset (3-clusters dataset), $\{\mathrm{Sil} / N C \mid P \in[12,17]\}$ is the stable space

Figure 3. The effect of input preference $(P)$ on $N C$ and $10^{*} \operatorname{Sil}(10$ times Silhouette is set to emphasize its changing trend).

\section{Effect of damping factor on Silhouette index}

After finding the stable space between $\mathrm{Sil}$ and $\mathrm{NC}$, one can obtain better $P$ area. Next, finding optimal $P$ is a key issue of the proposed algorithm.

In AP algorithm, damping factor is set to avoid numerical oscillation. $\lambda$ is reduced from 0.9 to 0.5 as it is shown in Figure 4, Sil changes but high $\mathrm{Sil}$ is more stable and accurate. If different
$P$ values have the same $\mathrm{Sil}, \lambda$ is reduced and the $P$ corresponding to higher Sil is chosen, this method can be called " $\lambda$ test". The details of whole constraint rules-based search algorithm are as follows:

\section{Constraint Rules-Based Search Algorithm (CRS)}

In the CRAP algorithm, the parameter search starts by a large $P$ value, $P_{\max }=100$, then it is divided into ten isometric spaces, the $i$-th isometric space $\quad S_{i}=\left[(i-1) P_{\max } / 10, i P_{\max } / 10\right], \quad i \in[1,10]$, $\lambda=0.5$, and $P_{j}(j=[1,10])$ is used to represent each $P$ value in $S_{i}$. For each $S_{i}$, if $P_{j}$ satisfies the rule $|S i l| P_{j}-S i l\left|P_{j+1}\right|<0.02$, Sil $>=0.2$, the change between $\left[\operatorname{Sil}\left|P_{j}, \operatorname{Sil}\right| P_{j+1}\right]$ is very slight. The isometric space $S_{i}$ is the stable space if this slight change occurs more than twice. After finding the stable space $S_{i}$ in $P$-Sil plot, one can obtain better $P$ area. Next, finding optimal $P$ value in this $P$ area is a key issue.

Generally speaking, the best $P$ value will generate the best Sil. In the case of one-to-one mapping (each Sil has one corresponding different $P$ value), the $P$ value is obtained when AP gets the largest $\mathrm{Sil}$ in the stable space. However, AP may obtain same $S i l$ with different $P$ values (many-to-one mapping), so damping factor (another parameter in AP algorithm) is used to decide which $P$ value is appropriate. When $\lambda$ is reduced from 0.9 to 0.5 as it is shown in Figure 4, Sil also changes. When $\lambda=0.9$, their results are identical, one adjusts $\lambda_{\text {step }}=-0.1, \quad S_{i l}(p=25, \lambda=0.8)>S_{i l}(p=24, \lambda=0.8)$, $P=25$ is the best value for Wine dataset. Based on this process, a more stable and accurate $P$ value will be chosen. This process is called $\lambda$ test.

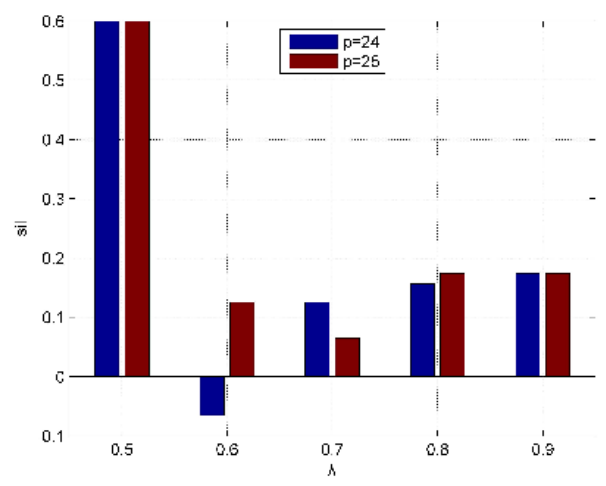

Figure 4. The process of $\lambda$ test using Wine dataset 
In brief, two kinds of strategies under different conditions are proposed to find the optimal $P$ value after obtaining the stable space: (1) if the relationship between $P$ and $\mathrm{Sil}$ is one-toone mapping, the $P$ value is obtained when the corresponding AP gets the largest $\mathrm{Sil}$ in the stable space. (2) if the relationship between $P$ and $S i l$ is many-to-one mapping, the $\lambda$ test is used to find the better $P$ value. The pseudo-codes of constraint rules-based search (CRS) algorithm are listed in Table 2.

Table 2. The pseudo-code of constraint rules-based search (CRS) algorithm

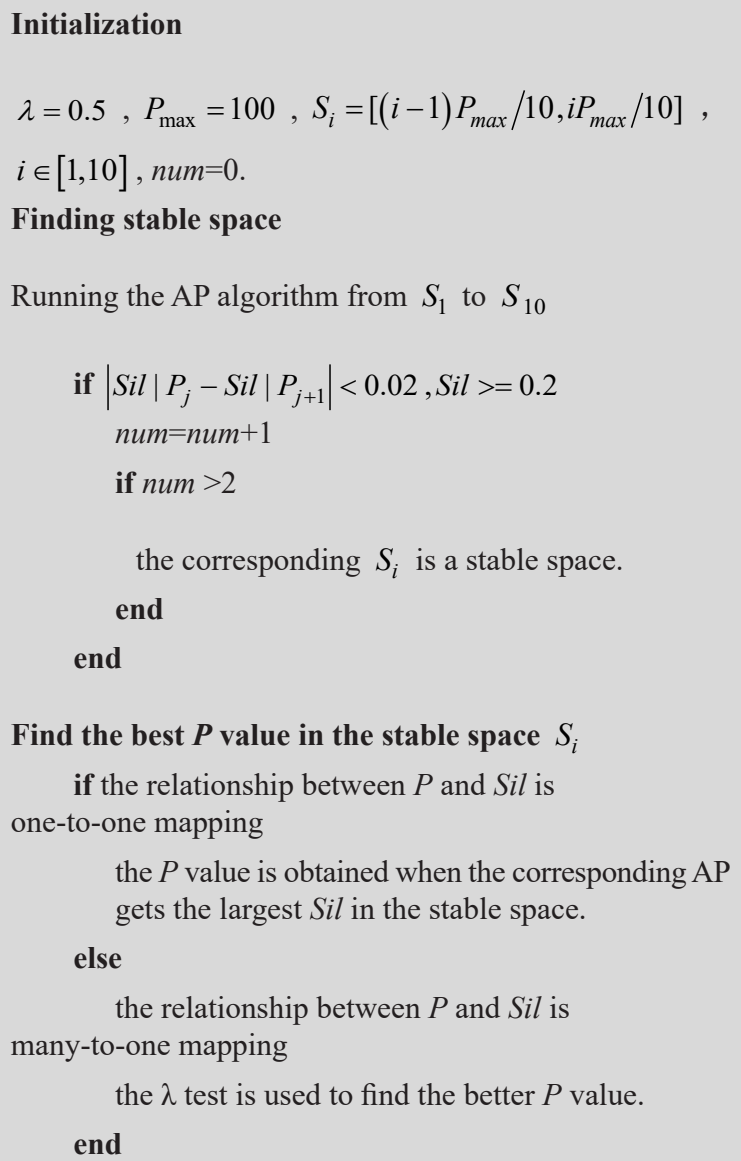

\section{Matching Micro-clusters Hierarchical Clustering Algorithm (MMHC)}

Clustering complex structure data is an important research field (Rodriguez \& Laio, 2014; Wang et al., 2020). The AP is unable to identify clusters with complex structure. The aim of this idea is to utilize advantages of AP to solve this problem.
Complex structure data has many topological structures, like manifold or non-manifold structure, uniform density or multiple density, overlapping and so on, MMHC mainly deals with structure of obvious boundaries between different clusters.

MMHC starts by an undirected graph $G=(V, E)$ like that in Figure 5 based on the micro-clusters generated by AP, each cluster $C_{i}$ has one path linking all the data points, $V$ denotes vertices (data points) and $E$ denotes edge (similarity between two data points).

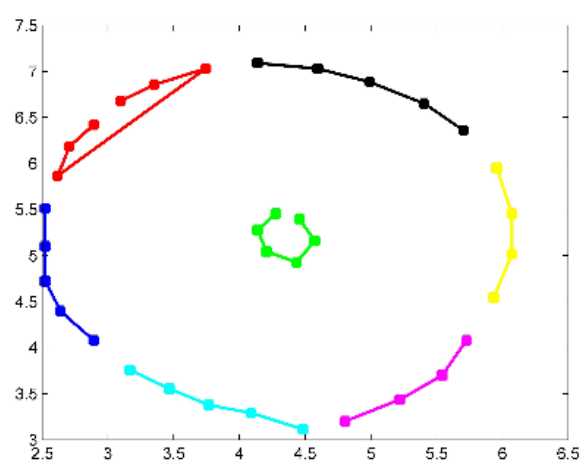

Figure 5. Undirected graph

MMHC has two important variables: inter-class density $(I C D)$ is defined as Equation (7), which describes compactness of a cluster.

$I C D=\min \left\{E \in C_{i}\right\}$

Intra-class topological distance (ICTD) is defined as Equation (8), which describes the gap between different clusters in the complex structure data:

$\left\{E_{\max } \in \max \left\{E \in C_{i}\right\}\right.$

$\left\{I C T D=\min \left\{S\left(\left(V \in E_{\max }\right), l\right)\right\}-I C D \quad l \in\left\{C_{i+1}\right\}\right.$

where the $S\left(\left(V \in E_{\max }\right), l\right)$ means Euclidean distance between data points $V \in E_{\max }$ and $l \in\left\{C_{i+1}\right\}, S\left(\left(V \in E_{\max }\right), l\right)=\|V-l\|$.

When the ICTD value is negative, the two clusters $C_{i}$ and $C_{i+1}$ are merged. Then the next microcluster becomes new $C_{i}$ and repeats the above merging process until all the micro-clusters are merged. The pseudo-codes of Micro-clusters Merging-based Clustering (MMHC) algorithm are listed in Table 3. 
Table 3. Procedure of Micro-clusters Merging-based Clustering (MMHC) algorithm

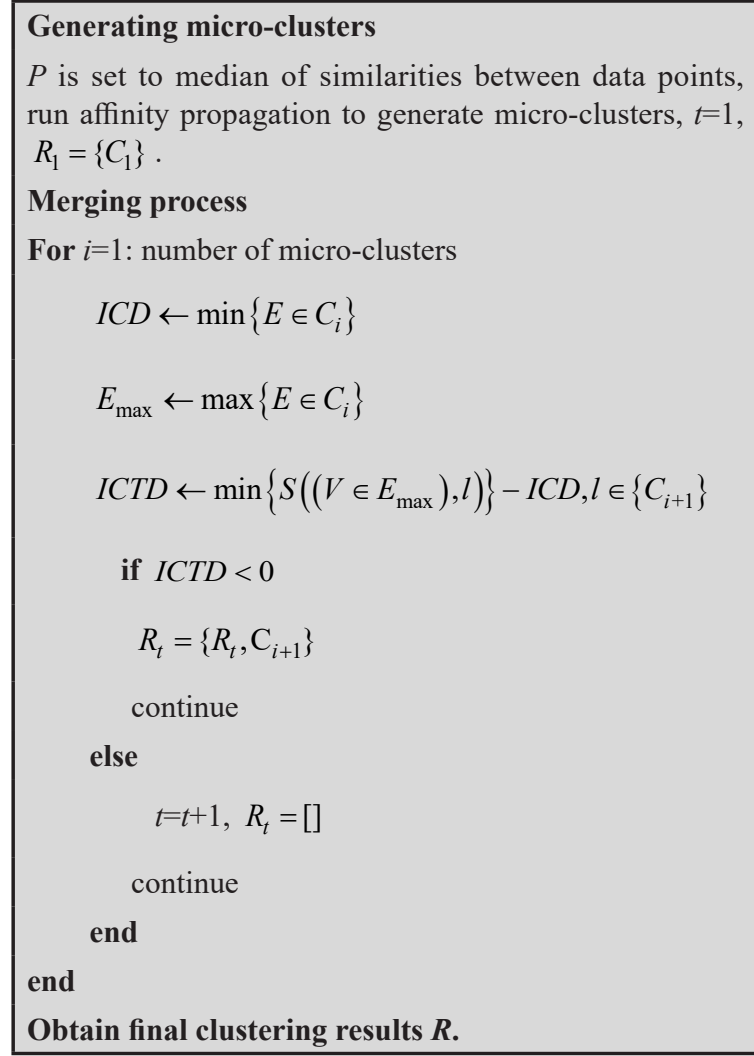

\section{Experimental Results and Analysis}

This section compares the performance of CRAP, MMHC with AP algorithm. To validate the effectiveness of the proposed methods, one uses same datasets, same machine to make experiments. All the experiments use $\lambda=0.5$ (but $\lambda$ changes in $\lambda$ test) and same hardware devices (Pentium G645 2.9 GHz CPU, 4GB memory), CRAP uses $P_{\max }=100$.

Table 4. Features of datasets.

\begin{tabular}{|c|c|c|c|}
\hline Data Sets & $\begin{array}{c}\text { Number of } \\
\text { samples }\end{array}$ & Dimensions & Source \\
\hline Wine & 178 & 13 & UCI \\
\hline Iris & 150 & 4 & UCI \\
\hline Seeds & 210 & 7 & UCI \\
\hline Harberman & 306 & 3 & UCI \\
\hline Jain & 373 & 2 & $\begin{array}{c}\text { (Rodriguez \& } \\
\text { Laio, 2014) }\end{array}$ \\
\hline Spiral & 312 & 2 & $\begin{array}{c}\text { (Rodriguez \& } \\
\text { Laio, 2014) }\end{array}$ \\
\hline 2circle & 35 & 2 & $\begin{array}{c}\text { (Wang et al, } \\
2020 \text { ) }\end{array}$ \\
\hline
\end{tabular}

The clustering results of CRAP and AP are listed in Table 5. For all the datasets, CRAP performs better than AP in term of most evaluation metrics.

Table 5. Clustering results of CRAP and AP

\begin{tabular}{|c|c|c|c|c|c|}
\hline Data sets & $\begin{array}{c}\text { CRAP } \\
\text { sil }\end{array}$ & $\begin{array}{c}\text { CRAP } \\
N C\end{array}$ & $\begin{array}{c}\mathrm{AP} \\
\text { sil }\end{array}$ & $\begin{array}{c}\mathrm{AP} \\
N C\end{array}$ & $\begin{array}{c}\text { Known } \\
N C\end{array}$ \\
\hline Wine & 0.5996 & 3 & -0.3544 & 12 & 3 \\
\hline Iris & 0.5057 & 3 & -0.1894 & 12 & 3 \\
\hline Seeds & 0.4325 & 3 & -0.2594 & 17 & 3 \\
\hline Harberman & 0.4099 & 2 & -0.2668 & 31 & 2 \\
\hline
\end{tabular}

Figure 6 shows clustering results of CRAP and AP using synthetic dataset, $\operatorname{Sil}(\mathrm{AP})=-0.3282, N C$ is 7 . $\operatorname{Sil}($ CRAP $)=0.3991, N C$ is 2 . Visually, clustering this synthetic dataset into 2 clusters is more reasonable than 7 clusters. Meantime, $\operatorname{Sil}(\mathrm{CRAP})>$ Sil(AP). These results demonstrate that the constraint rules and search techniques in CRAP method are effective, resulting in better performance of CRAP in comparison with original AP.

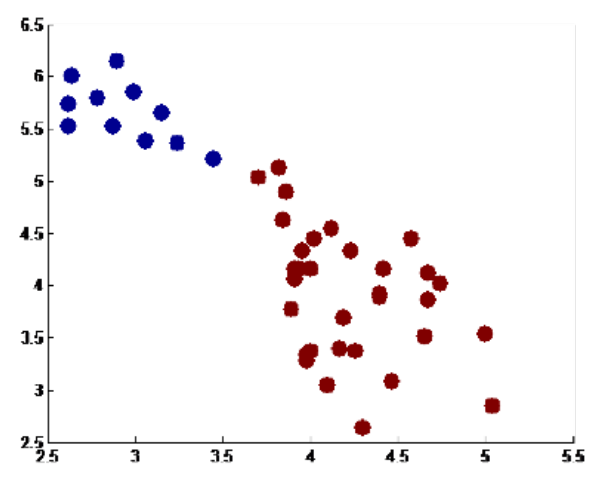

(a) CRAP

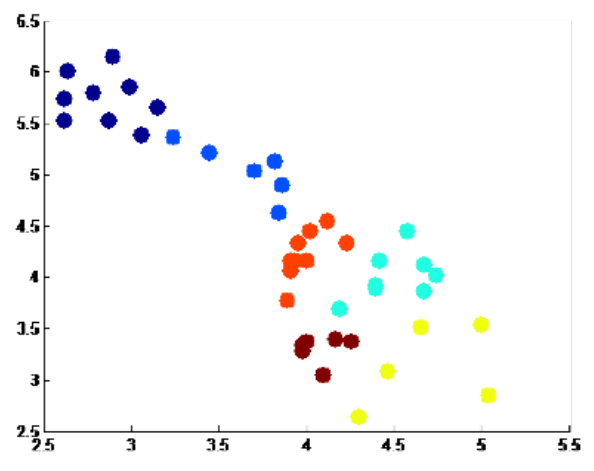

(b) AP

Figure 6. Clustering results of CRAP and AP on a synthetic dataset, which has two-clusters in ground-truth 
Experiments are also carried out to verify the effectiveness of the MMHC algorithm by contained, occlusive and other complex structure of obvious boundaries. As it is shown in Figure (7), (8) and (9), compared with AP and CRAP, the MMHC algorithm can clearly identify the clusters with complex structure, while AP and CRAP cannot. AP and CRAP are based on Euclidean similarities, which cannot accurately identify complex structure.

The proposed methods have their own strengths and weaknesses, if data has no stable space in Sil/ $N C-P$ plot, CRAP cannot obtain optimal NC. This paper also proposes MMHC algorithm to identify clusters with complex structure by merging microclusters generated by AP.

\section{Application in Chinese Stock Quality Assessment}

The method proposed in this paper can also be applied to Chinese stock quality assessment. Financial indicators of 98 listed companies are taken as model input, financial indicators include earnings per share (EPS), capital reserve per share (CRPS), undistributed profit per share (UPPS), return on equity (ROE) and net assets per share (NAPS). 98 listed companies are merged into two classes by MMHC algorithm, which include 31 and

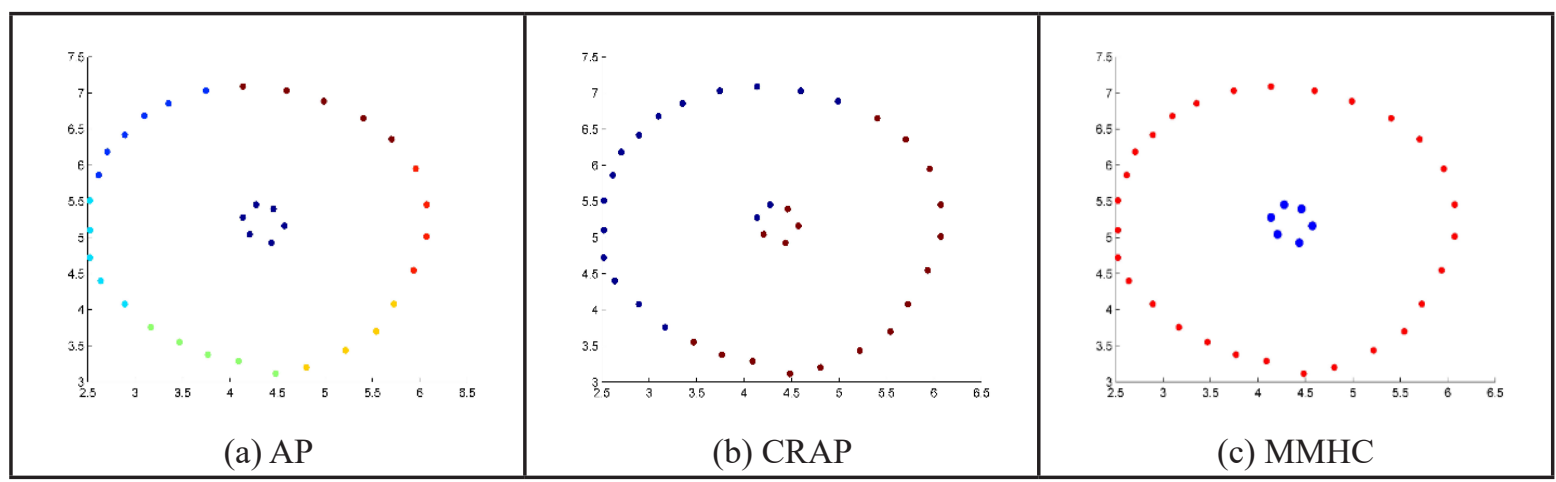

Figure 7. Clustering results of AP, CRAP and MMHC using 2circle dataset

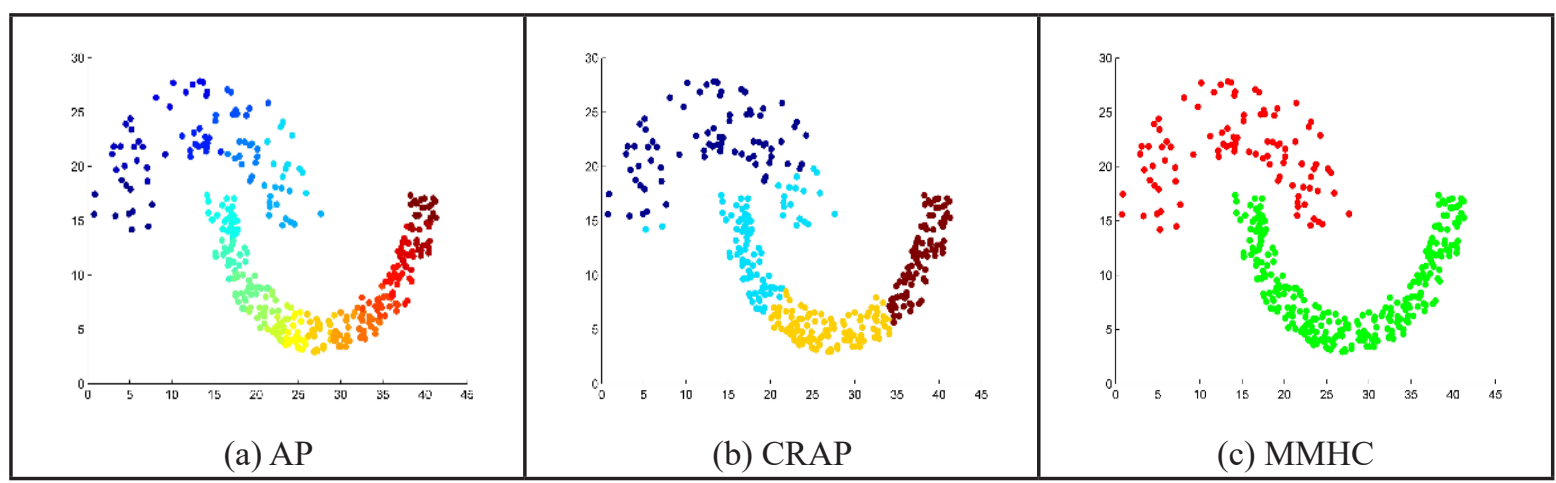

Figure 8. Clustering results of AP, CRAP and MMHC using Jain dataset

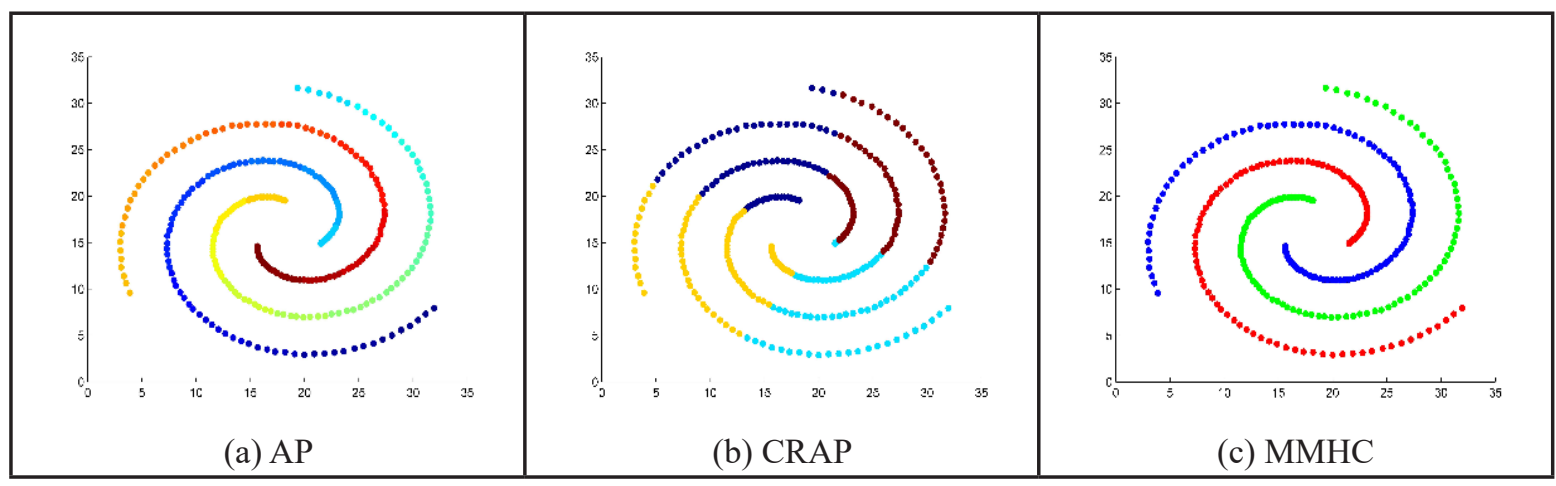

Figure 9. Clustering results of AP, CRAP and MMHC using Spiral dataset 
67 listed companies respectively. The statistical data related to the financial indicators of two types of listed companies is shown in the Table 6 .

Table 6. Statistical data of financial indicators of two types of listed companies obtained by MMHC

\begin{tabular}{|c|c|c|c|c|c|}
\hline Results & EPS* & CRPS* & UPPS* & ROE* & NAPS* \\
\hline $\begin{array}{c}\text { Class } \\
1(31)\end{array}$ & 0.4864 & 0.816 & 2.247 & 12.863 & 4.45 \\
\hline $\begin{array}{c}\text { Class } \\
2(67)\end{array}$ & 0.0742 & 0.879 & 1.084 & 0.084 & 3.161 \\
\hline
\end{tabular}

The average earnings per share of the class 1 is 6.55 times that of the second category, and the average return on net assets of the first category is 7.62 times that of the second category. There are obvious differences between these two important financial indicators, which shows that the MMHC algorithm has a significant recognition of the unknown real class data, strong practicability, and can effectively distinguish the status of enterprises in the industry.

Table 7. Statistical data of financial indicators of two types of listed companies obtained by AP

\begin{tabular}{|c|c|c|c|c|c|}
\hline Results & EPS* & CRPS* & UPPS* & ROE* & NAPS* \\
\hline $\begin{array}{c}\text { Class } \\
1(28)\end{array}$ & 0.4953 & 0.851 & 2.357 & 12.936 & 4.86 \\
\hline $\begin{array}{c}\text { Class } \\
2(70)\end{array}$ & 0.0883 & 0.8623 & 1.090 & 0.6024 & 3.052 \\
\hline
\end{tabular}

98 listed companies have no labels, the popular clustering evaluation indexes are useless. Thus, Mean Square Error (MSE) is used to evaluate the quality of two kinds of methods. The larger the MSE is, the better the method is. A larger MSE means the clustering results are better. Obviously,
MSE of AP is 31.43 and MSE of MMHC is 33.298, which reveals that the proposed method is better than original AP.

\section{Conclusion}

This paper presents new approaches to obtain the optimal $P$ and achieve complex structure clustering. A reasonable number of real-world and synthetic datasets are used to verify the proposed CRAP and MMHC algorithms, which perform significantly better than the original AP. The MMHC can combine with many algorithms to realize complex structure clustering. On the other hand, there are also problems with regard to CRAP and MMHC algorithms. Although one can deal with datasets of 2circle, Jain and Spiral, other structures without line between different clusters are unsuited for MMHC, further research is needed.

\section{Acknowledgements}

This research is supported by the National Science Foundation of China under Grant No. 61572225 and 61472049, the foundation of Jilin Province Education Department under Grant No. JJKH20190724KJ, the Jilin Provincial Science \& Technology Department Foundation under Grant No. 20190302071GX and 20200201164JC, and the Development and Reform Commission Foundation of Jilin Province under Grant No. 2019C053-11. This research is also supported by the National Natural Science Foundation of China (Grants No. 61772227, 61972174, 61972175), Science and Technology Development Foundation of Jilin Province (No. 20180201045GX, 20180414012GH), and the Jilin Development and Reform Commission Fund (No. 2020C020-2).

\section{REFERENCES}

Angelova, M., Beliakov, G. \& Zhu, Y. (2019). Density-based clustering using approximate natural neighbours, Applied Soft Computing, 85, 1-10. Article ID: 105867 .

Darabkh, K. A., Odetallah, S. M., Al-qudah, Z. \& Khalifeh, A. (2019). Energy-aware and density-based clustering and relaying protocol (EA-DB-CRP) for gathering data in wireless sensor networks, Applied Soft Computing, 80, 154-166.

Dutta, A. K., Elhoseny, M., Dahiya, V. \& Shankar, K. (2020). An efficient hierarchical clustering protocol for multihop Internet of vehicles communication, Transactions on Emerging Telecommunications Technologies, 31(5): e3690. DOI: 10.1002/ett.3690
Fan, Z., Jiang, J., Weng, S., He, Z. \& Liu, Z. (2019). Adaptive density distribution inspired affinity propagation clustering, Neural Computing and Applications, 31(1), 435-445.

Fard, M. M., Thonet, T. \& Gaussier, E. (2020). Deep k-means: Jointly clustering with k-means and learning representations, Pattern Recognition Letters, 1-5. DOI: $10.1016 /$ j.patrec.2020.07.028

Feldman, D., Schmidt, M. \& Sohler, C. (2020). Turning big data into tiny data: Constant-size coresets for k-means, PCA, and projective clustering, SIAM Journal on Computing, 49(3), 601-657. 
Frey, B. J. \& Dueck, D. (2007). Clustering by passing messages between data points, Science, 315(5814), 972-976.

Gao, Q., Wang, Y., Cheng, X., Yu, J., Chen, X. \& Tao, J. (2019). Identification of Vulnerable Lines in Smart Grid Systems Based on Affinity Propagation Clustering, IEEE Internet of Things Journal, 6(3), 5163-5171.

Givoni, I., Chung, C. \& Frey, B. J. (2012). Hierarchical Affinity Propagation, Psi.toronto.edu, 1-5.

Hassanabadi, B., Shea, C., Zhang, L., Valaee, S. (2014). Clustering in vehicular ad hoc networks using affinity propagation, Ad Hoc Networks, 13(B), 535-548.

Li, P., Ji, H., Wang, B., Huang, Z. \& Li, H. (2017). Adjustable preference affinity propagation clustering, Pattern Recognition Letters, 85, 72-78.

Li, X., Zhang, L., Wang, L. \& Wan, X. (2017). Effects of BOW model with affinity propagation and spatial pyramid matching on polarimetric SAR image classification, IEEE Journal of Selected Topics in Applied Earth Observations and Remote Sensing, 10(7), 3314-3322.

Lu, Z. \& Carreira-Perpinan, M. A. (2008). Constrained spectral clustering through affinity propagation. In 2008 IEEE Computer Society Conference on Computer Vision and Pattern Recognition (CVPR 2008), (pp. 1-8).

Qasim, I., Jeong, J.-W., Heu, J.-U. \& Lee, D.-H. (2013). Concept map construction from text documents using affinity propagation, Journal of Information Science, 39(6), 719-736.

Rodriguez, A. \& Laio, A. (2014). Clustering by fast search and find of density peaks, Science, 344(6191), 1492-1496. DOI: 10.1126/science. 1242072

Samaria, F. S. \& Harter, A C. (1994). Parameterisation of a stochastic model for human face identification. In Proceedings of 1994 IEEE Workshop on Applications of Computer Vision (pp. 138-142).

Sun, L., Guo, C., Liu, C. \& Xiong, H. (2017). Fast affinity propagation clustering based on incomplete similarity matrix, Knowledge and Information Systems, 51(3), 941-963.

Sun, Y., Xie, Z. \& Gao, J. (2013). A Novel Kernel Classification Method via Image Novelty Detection, Acta Optica Sinica, 33(10), 1256-1272.

Wang, Y., Wang, D., Pang, W., Miao, C., Tan, A.-H. \& Zhou, Y. (2020). A Systematic Densitybased Clustering Method Using Anchor Points, Neurocomputing, 400, 352-370.

Wang, Z. J., Zhan, Z.-H., Lin, Y., Yu, W.-J., Yuan, H., Gu, T., Kwong, S. \& Zhang, J. (2017). Dual-strategy differential evolution with affinity propagation clustering for multimodal optimization problems, IEEE Transactions on Evolutionary Computation, 22(6), 894-908.

Wei, Z., Wang, Y., He, S. \& Bao, J. (2017). A novel intelligent method for bearing fault diagnosis based on affinity propagation clustering and adaptive feature selection, Knowledge-Based Systems, 116, 1-12.

Zhang, J., Chen, F.-C., Shao-Mei, L. \& Liu, L.-X. (2014). Data Stream Clustering Algorithm Based on Density and Affinity Propagation Technique, Acta Automatica Sinica, 40(2), 277-288.

Zhang, X., Furtlehner, C., Germain-Renaud, C \& Sebag, M. (2014). Data stream clustering with affinity propagation, IEEE Transactions on Knowledge and Data Engineering, 26(7), 1644-1656.

Zhang, L. M., Jia, Y., Zhou, B. \& Zhao, J.-H. (2013). Online bursty events detection based on emoticons, Chinese Journal of Computers, 36(8), 1659-1667.

Zhang, Z., Wang, B.-Q. \& Li, X.-T. (2013). Semisupervised Traffic Identification Based on Affinity Propagation, Acta Automatica Sinica, 39(7), 1100-1109.

Zhang, W., Wu, X., Zhu, W.-P. \& Yu, L. (2017). Unsupervised image clustering with SIFT-based softmatching affinity propagation, IEEE Signal Processing Letters, 24(4), 461-464. 